\title{
SOME PROPERTIES OF CONTINUOUS CURVES*
}

BY G. T. WHYBURN

The points $A$ and $B$ of a continuum $M$ are said to be separated in $M$ by a point $X$ of $M$ if $M-X$ is the sum of two mutually separated sets $S_{1}$ and $S_{2}$ containing $A$ and $B$ respectively. The point $P$ of a continuum $M$ is a cut point of $M$ if and only if the set of points $M-P$ is not connected, i.e., is the sum of two mutually separated point sets.

A continuous curve $M$ will be said to be cyclicly connected provided that every two points of $M$ lie together on some simple closed curve which is contained in $M$. In this paper use will be made of the following fundamental theorem.

THEOREM A. In order that the continuous curve $M$ should be cyclicly connected it is necessary and sufficient that $M$ should have no cut point.

A proof for Theorem A will be found in my paper Cyclicly connected continuous curves, which will appear soon.

Theorem I. If $A$ and $B$ are any two points of a continuous curve $M$ and if $K$ denotes the set of all those points of $M$ which separate $A$ from $B$ in $M$, then $K+A+B$ is a closed set of points.

Proof. The curve $M$ contains a simple continuous arc $t$ from $A$ to $B$. Clearly $K$ must be a subset of $t$. Let $P$ be any point of $t-(K+A+B)$. Since $P$ does not belong to $K$, $A$ and $B$ must both belong to some connected subset $\dagger$ of $M-P$, and by a theorem of $\mathrm{R}$. L. Moore'st it follows that $M-P$ contains an arc $t^{\prime}$ from $A$ to $B$. On the $\operatorname{arcs} P A$

* Presented to the Society, December 31, 1926.

† See an abstract of a paper by R. L. Wilder, $A$ characterization of continuous curves by a property of their open subsets, this Bulletin, vol. 32 (1926), pp. 217-218.

$\ddagger$ Concerning continuous curves in the plane, Mathematische Zeitschrift, vol. 15 (1922), p. 255. 
and $P B$ of $t$, in the order from $P$ to $A$ and from $P$ to $B$ respectively, let $X$ and $Y$ respectively denote the first points belonging to $t^{\prime}$. Then no point of the segment $X P Y$ of $t$ can belong to $K+A+B$. Since thus every point of $t-(K+A+B)$ belongs to some segment of $t$ which contains no point of $K+A+B$, the set $K+A+B$ is closed.

Definition. A cyclicly connected continuous curve $C$ is said to be a maximal cyclic curve of a continuous curve $M$ if $C$ is a subset of $M$ and is not a proper subset of any other cyclicly connected continuous curve belonging to $M$.

Theorem II. If $A$ and $B$ are any two points of a continuous curve $M, t$ is any arc of $M$ from $A$ to $B, K$ denotes the set of all those points of $M$ which separate $A$ from $B$ in $M$, and $S$ is any maximal segment of $t-(K+A+B)$, then $M$ contains a maximal cyclic curve which contains $S$.

Proof. Let $E$ and $F$ denote the end points of $S$. Let $G$ denote the collection of all the maximal connected subsets of $M-(E+F)$. At least one element of $G$, namely, the one which contains $S$, must have both of the points $E$ and $F$ for limit points. Only a finite number of elements of $G$ can have this property. Let $H$ denote the point set obtained by adding together all the elements of $G$ which do have this property, and let $N$ denote the point set $H+E+F$. Then $N$ is closed and connected. The continuum $N$ is also connected im kleinen. For since $M$ is connected im kleinen, no point of $H$ is a limit point of $M-H$. Hence $H$ is connected im kleinen. Then from a theorem of R. L. Moore's* it easily follows that $H+E+F=N$ must also be connected im kleinen. Thus we see that $N$ is a continuous curve.

Now if $N$ has no cut point, then by Theorem A, $N$ must be cyclicly connected. If $N$ has any cut points, then for every cut point $X$ of $N$, let $H_{x}$ denote the maximal connected subset of $N-X$ which contains $(S+X)-X$, and let $N_{x}$ denote the point set $H_{x}+X$. That $H_{x}$ exists in case $X$ is

* $A$ report on continuous curves from the viewpoint of analysis situs, this Bulletin, vol. 29 (1923), pp. 296-297. 
not on $S$ is obvious. If $X$ belongs to $S$, then since $X$ does not belong to $K+A+B, M-X$ contains an $\operatorname{arc}^{*} t^{\prime}$ from $A$ to $B$. The arc $t^{\prime}$ must contain the points $E$ and $F$, for $E$ and $F$ belong to $K+A+B$. The arc $E F$ of $t^{\prime}$ must belong to $N$, hence also to $N-X$. Therefore, $S-X$, being the sum of the segments $E X$ and $E F$ of $t$, must lie in some connected subset of $N-X$. Thus, in any case, $H_{x}$ exists. Now let $L$ denote the set of all points which are common to all the point sets $N_{x}$. Clearly $L$ exists, is a closed point set, and contains $S$. Let $C$ denote the maximal connected subset of $L$ which contains $S$. I shall show that $C$ is a maximal cyclic curve of $M$.

Clearly $C$ is closed and connected. I shall first show that if $P$ and $Q$ are any two points of $C$ and $P O Q$ is any arc of $N$ from $P$ to $Q$, then every point of $P O Q$ must belong to $C$. This must be true, for if $X$ is any cut point of $N$ not on $P O Q$, then $H_{x}$ contains every point of $P O Q$ because it contains $P$ and $Q$. And if $X$ is any cut point of $N$ on $P O Q$, then $H_{x}$ contains $P O Q-X$, because $P O Q-X$ is either a single connected set containing a point of $H_{x}$ or the sum of two connected sets each of which contains a point of $H_{x}$. Hence, in any case, $P O Q$ belongs to every set $N_{x}$ and therefore belongs to $L$ and to $C$.

The continuum $C$ is a continuous curve. For let $P$ be any point of $C$ and $\epsilon$ any positive number. Then since $N$ is connected im kleinen, there exists a positive number $\delta_{\mathrm{e}}$ such that every point of $N$ whose distance from $P$ is less than $\delta_{\mathrm{e}}$ can be joined in $N$ to $P$ by an arc which is of diameter less than $\epsilon$. Let $X$ be any point of $C$ whose distance from $P$ is less than $\delta_{\epsilon}$. Then $N$ contains an arc $a$ from $X$ to $P$ of diameter less than $\epsilon$. But as was shown above, $a$ must belong to $C$. Hence $C$ is connected im kleinen at every one of its points and is therefore a continuous curve.

The curve $C$ has no cut point. For suppose, on the contrary, that $C$ has a cut point $X$. Then $C-X=S_{1}+S_{2}$,

* See R. L. Wilder, loc. cit., and R. L. Moore, Concerning continuous curves in the plane, loc. cit. 
where $S_{1}$ and $S_{2}$ are mutually separated point sets. Let $P_{1}$ and $P_{2}$ be points of $S_{1}$ and $S_{2}$ respectively. Now if $X$ is a cut point of $N$, then since $H_{x}$ contains $P_{1}$ and $P_{2}$, it follows* that $N-X$ contains an arc $s$ from $P_{1}$ to $P_{2}$. And if $X$ is not a cut point of $N$, again it follows that $N-X$ contains an arc $s$ from $P_{1}$ to $P_{2}$. And in either case, as was shown above, the arc $s$ must belong to $C$. Hence $S_{1}$ and $S_{2}$ are not mutually separated, contrary to supposition. Therefore $C$ has no cut point, and by Theorem $\mathrm{A}$ it follows that $C$ is a cyclicly connected continuous curve. That $C$ is a maximal cyclic curve of $M$ follows immediately from the facts (1) that every arc of $M$ joining two points of $N$ must belong wholly to $N$ and (2) that every arc of $N$ joining two points of $C$ must belong wholly to $C$. This completes the proof.

Theorem III. If $A$ and $B$ are any two points of a continuous curve $M$ and if $K$ denotes the set of all those points of $M$ which separate $A$ from $B$ in $M$, then $M$ contains two simple continuous arcs $t_{1}$ and $t_{2}$ from $A$ to $B$ whose common part is $K+A+B$.

Proof. By Theorem I, $K+A+B$ is a closed set of points. Hence if $t$ is any arc of $M$ from $A$ to $B, t$ must contain $K$, and $t-(K+A+B)$ is the sum of a countable number of non-overlapping segments $S_{1}, S_{2}, S_{3}, \cdots$. By Theorem II it follows that for every positive integer $i, M$ contains a maximal cyclic curve $C_{i}$ which contains $S_{i}$. For each $i$, let the end points of $S_{i}$ be denoted by $A_{i}$ and $B_{i}$. Since $C_{i}$ is cyclicly connected, then for each $i, C_{i}$ contains two arcs $t_{1 i}$ and $t_{2 i}$ from $A_{i}$ to $B_{i}$ whose common part is only the points $A_{i}$ and $B_{i}$. Let

$$
t_{1}=K+A+B+\sum_{i=1,2,3, \cdots} t_{1 i}, \quad t_{2}=K+A+B+\sum_{i=1,2,3} t_{2 i} .
$$

Then $t_{1}$ and $t_{2}$ are simple continuous arcs of $M$ from $A$ to $B$ whose common part is $K+A+B$.

ThE UNIVERsity OF TeXas

\footnotetext{
${ }^{*}$ See R. L. Moore, loc. cit.
} 